\title{
Société suisse des médecins-conseils SSMC
}

Jürg Zollikofer

Président de la SSMC

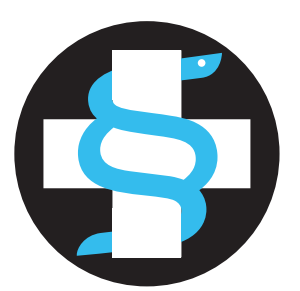

Correspondance: Dr Jürg Zollikofer Im Zinggen 1 $\mathrm{CH}-8475$ Ossingen juerg.zollikofer@hin.ch

La Société suisse des médecins-conseils (SSMC) est la société de discipline de la FMH qui regroupe les médecins d'assurance. Ses membres sont exclusivement des médecins s'occupant de questions relevant de la médecine des assurances. A l'origine un groupement de médecinsconseils travaillant pour des caisses-maladie, la SSMC s'est développée ces der-

SGV SSMC

Schweizerische Gesellschaft der Vertrauensärzte

Société Suisse des Médecins-Conseils Società Svizzera Società Svizzera
Medici Fiduciari nières années en une association englobant l'ensemble de la médecine des assurances. Elle s'occupe de toutes les questions de politique sanitaire présentant des aspects de médecine des assurances, sur le plan notamment des frais de guérison et de l'évaluation des risques. Sur mandat de la FMH et de santésuisse, la SSMC organise des cours de formation postgraduée pour l'attestation de formation complémentaire «médecinconseil», en allemand à Winterthour et en français à l'Université de Fribourg. Plus de 260 attestations de formation complémentaire ont été délivrées jusqu'à présent.

Conformément à sa ligne directrice, la SSMC est une force active dans l'élaboration de recommandations en médecine des assurances. Ses compétences spécialisées lui permettent de jouer un rôle de leader et de participer aux décisions concernant toutes les questions de ce domaine. Sa position dans le système de santé publique correspond au statut d'une société de discipline. Elle se présente activement en cette qualité auprès de la FMH, des partenaires et du public. La médecine des assurances est la synthèse entre des faits médicaux et les lois et ordonnances applicables en la matière, avec prise en compte des progrès médicaux, des données économiques et des principes éthiques.

La Commission d'experts élabore des bases fondées sur des preuves pour les questions de médecine des assurances. La Commission pour la formation postgraduée et continue veille à offrir une formation permanente, compétente et scientifiquement reconnue.

La SSMC gère un site internet (www.medecinsconseils.ch ou www.vertrauensaerzte.ch), où l'on peut trouver facilement, entre autres, des recommandations de sa Commission d'experts ainsi que l'ensemble des lois et des ordonnances. Dans la partie réservée à ses membres, elle met à leur disposition un forum de discussions et d'échange.

Les membres de la SSMC - médecins-conseils des assureurs-maladie, médecins de la Suva, des services médicaux régionaux, des centres d'observation médicale ou médecins consultants auprès d'assureurs privés, d'entreprises et d'autorités - se considèrent avant tout comme médecins et, partant, comme intermédiaires entre assureurs, fournisseurs de prestations et patients. Néanmoins, ils sont souvent perçus comme les défenseurs de la partie adverse: de l'assureur selon les fournisseurs de prestations et du fournisseur de prestations selon les assureurs.

La SSMC publie le «Manuel des médecinsconseils». Elle mène actuellement des travaux en vue de la $3^{\text {e }}$ édition. Ce manuel rend service à toute personne travaillant dans le domaine des assurances sociales.

Parmi les tâches incombant à la SSMC figure l'organisation du congrès annuel dont le prochain aura lieu dès le 16 avril 2008 à Fribourg. Des ateliers seront offerts sur différents sujets. Le $2^{\mathrm{e}}$ jour est consacré à un thème particulier; les discussions porteront ainsi le 17 avril sur des questions relatives à la protection des données. Outre des représentants d'organisations de patients et d'assureurs, la SSMC recevra les orateurs suivants: Hanspeter Thür, préposé fédéral à la protection des données et à la transparence, Rudolf Luginbühl, ombudsman des assureursmaladie, et Jacques de Haller, président de la FMH. Les conférences sont publiques. Vous trouverez de plus amples informations sous: www.medecins-conseils.ch. 